\title{
Development of EIS cell chips and their application for cell analysis
}

\author{
Elisabetta Primiceri *, Maria Serena Chiriacò, Rodica Elena Ionescu, Eliana D’Amone, Roberto Cingolani, \\ Ross Rinaldi, Giuseppe Maruccio
}

National Nanotechnology Lab of CNR-INFM, Via per Arnesano Km4, 73100 Lecce, Italy

\section{A R T I C L E I N F O}

Article history:

Received 30 September 2008

Received in revised form 20 November 2008

Accepted 10 December 2008

Available online 24 December 2008

Keywords:

Cell Chip

Impedance Spectroscopy

Cell counting

Lab on chip

Nanotechnology

\begin{abstract}
A B S T R A C T
We report the development of EIS cell chips able to monitor cell growth and adhesion. They are made of transparent or semitransparent materials to allow complementary analysis of cell behaviour during the measurements through optical microscopy. Our approach is cheap both in fabrication and usage, it is not invasive for cells and it does not require any additional reagent. Our devices are particular suitable to count cells or to evaluate cell morphology and changes as a consequence of different treatments.
\end{abstract}

(c) 2009 Published by Elsevier B.V.

\section{Introduction}

A new trend in the biosensor/lab-on-chip field is the development of cell-based devices for cell culture analysis, detection of specific analytes and/or testing of large libraries of substances (such as new drugs or potentially toxic compounds) for their biological effects. In this respect, electrochemical impedance spectroscopy (EIS) is a powerful tool for indirect studies about cells behaviour. In fact adhesion of cells on biofunctionalized electrodes alters the capacitance $C$ and interfacial electron transfer resistance $\mathrm{R}_{\mathrm{ET}}$ making EIS able to monitor real-time cell behaviour without any damage $[1,2]$. Specifically upon the attachment and spreading of cells onto the electrodes the impedance increases because a cell layer acts as an insulating film and $\mathrm{R}_{\mathrm{ET}}$ and $\mathrm{C}$ are correlated to cell viability, adhesion and cytoskeleton organization.

Thanks to recent progresses, applications cover a large part of cell biology, including detection of metabolic activity, cell adhesion and interaction with extracellular matrix protein [3], spreading [4]. In this respect, it is worth noting that so far the tools for studying adhesion and spreading are typically based on optical observation but they are time expensive and not quantitative. As a consequence, EIS devices are very interesting being (i) sensitive enough to discriminate the effect on cells of different proteins or different molecules on the surface and (ii) easily downscalable and suitable to be multiplexed at a very high level in dedicated chips (for example for parallel drug screening or cytotoxicity tests). To date, EIS has been successfully used for cytotoxicity tests on different cell

\footnotetext{
* Corresponding author. Tel.: +39 0832 298382; fax: +39 0832298386

E-mail address: elisabetta.primiceri@unile.it (E. Primiceri).
}

lines, such as human hepatocellular carcinoma cells [5], fibroblasts [6-8], cells from kidney of male monkey or human colon adenocarcinoma [9]. Even in this case the conventional biochemical methods currently used in cell biology are time consuming and laborious and require complex steps with multiple reagents. In addition, these techniques are invasive preventing to monitor a dynamic process in a single experiment. EIS is able to overcome these limitations because it is easier, faster and cheaper than conventional methods. Moreover, it is not only suitable to study adhering cells but also for cells in solution such as bacteria or blood cells [10] and it has been for example used for the detection of several pathogens such as Salmonella typhimurium and Escherichia coli $[11,12]$. Other applications include analysis of cells micromotion, cells attachment and spreading, cell concentration and growth, apoptosis and others [13-18]).

Here we describe the development of EIS cell chips able to monitor cell growth and adhesion. They are made of transparent or semitransparent materials to allow complementary analysis of cell behaviour during the measurements through optical microscopy (in bright field). Our approach is cheap both in fabrication and usage, it is not invasive for cells and it does not requires any additional reagent. These devices are particular suitable to count cells or to evaluate cell morphology and changes as a consequence of different treatments.

\section{Fabrication process and EIS mesurement}

Our chip (Fig. 1) consists of a cell culture chamber made of PDMS incorporating interdigitated electrodes fabricated on glass 

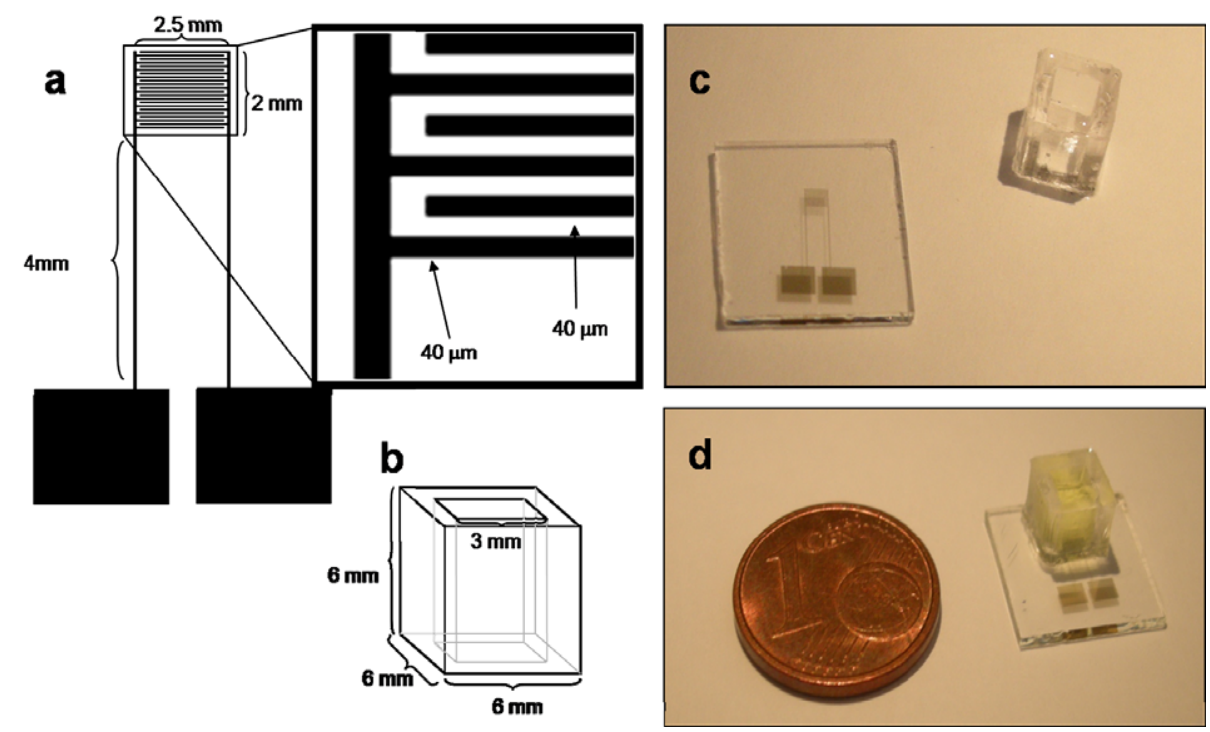

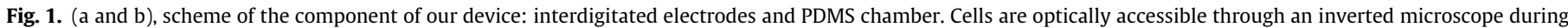
the measurements as shown in. $\mathrm{c}$ and $\mathrm{d}$ picture of the separate components (c) and of the assembled device (d).

substrates. For the conductive electrodes, we employed different materials: gold, ITO and platinum. In particular, ITO was used since it is a transparent material so the whole device is completely transparent in order to look at cells during the measurement through an inverted optical microscope (Fig. 1b). On the other hand, gold and platinum electrodes are more resistant and allow an easy functionalization (by thiol chemistry).

In the case of ITO electrodes, glass substrates with ITO layer (120 nm thick) were used (Visionteck). After cleaning with acetone and isopropanol, the electrode pattern was defined by means of a combination of optical lithography (AZ5214E photoresist, Karl Suss MJB3 mask aligner) and dry etching carried out with a RIE (Reactive Ion Etching) with a mixture of $\mathrm{CH}_{4} / \mathrm{H}_{2} / \mathrm{Ar}$ at $200 \mathrm{~W}$ for $30 \mathrm{~s}$. On the other hand, gold/platinum electrodes were fabricated by photolithography, thermal/e-beam deposition (respectively $3 \mathrm{~nm}$ of chromium/titanium and $10 \mathrm{~nm}$ of gold/platinum) and lift-off. In all cases the electrode pattern consists of interdigitated lines with a line-space period of $40 \mu \mathrm{m}$, covering an active area of $2 \times 2.5 \mathrm{~mm}^{2}$ (Fig. 1a).

PDMS cell culture chambers (Fig. 1b) were realized by replica molding from a hard master. We used a mixture of polymer base and curant agent $(15: 1 \mathrm{w} / \mathrm{w})$ and we induced the polymerization by heating at $140{ }^{\circ} \mathrm{C}$ for $10 \mathrm{~min}$. To improve the adhesion between the chamber and the glass substrate we have added at the bottom of the chamber a thin layer of PDMS with a ratio between polymer base and curant of 10:1 (w/w) and we have heated again for few minutes at $140^{\circ} \mathrm{C}$.

The whole device (Fig. 1d) is thus made using transparent (or semitransparent) and biocompatible materials in order to be mounted on an inverted microscope for real-time monitoring of cells during measurements.

An impedance analyzer Autolab PGSTAT30 (EcoChemie) with a FRA2 module was used for measurements. Impedance data were recorded in the frequency range between 1 and $10^{6} \mathrm{~Hz}$ using sinusoidal ac voltages (rms amplitude $15 \mathrm{mV}$ ). The dc bias potential between the two planar gold or ITO electrodes was clamped to $0 \mathrm{~V}$.

All the experiments were performed in milliQ water or L15 Medium (Leibovitz) from Sigma-Aldrich, a conventional cell culture medium formulated to be used in carbon dioxide free systems to maintain the cells outside the incubator during the measurement. A redox couple $\mathrm{K}_{3}\left[\mathrm{Fe}(\mathrm{CN})_{6}\right] / \mathrm{K}_{4}\left[\mathrm{Fe}(\mathrm{CN})_{6}\right]$ (1:1) (Sigma-Aldrich) was added to the medium to a final concentration ranging from 5 to $20 \mathrm{mM}$ to increase the conductivity of the solution and the sensitivity of the system but it is low enough to avoid any possible toxic effect on cells.

\section{Results and discussion}

Typically, EIS is carried out in water solution, but to work with cells it is necessary to use an appropriate culture medium containing all the nutrients and growth factors that have a key role for cells survival. So we firstly investigated the influence of the culture medium on our measurements by testing milliQ water added with $\mathrm{K}_{3}\left[\mathrm{Fe}(\mathrm{CN})_{6}\right] / \mathrm{K}_{4}\left[\mathrm{Fe}(\mathrm{CN})_{6}\right]$ as redox probe and $\mathrm{L} 15$ medium with the same concentration of redox couple (since milliQ water has a very low conductivity, a redox probe is necessary to improve the conductivity of the solution and the sensitivity of the system and was added at the same concentration in both water and L15 medium). Similar impedance profiles (Fig. 2) were obtained in both cases with a slightly higher modulus and smaller error bars in L15. According to this data L15 does not interact significantly with the redox couple $\mathrm{K}_{3}\left[\mathrm{Fe}(\mathrm{CN})_{6}\right] / \mathrm{K}_{4}\left[\mathrm{Fe}(\mathrm{CN})_{6}\right]$ and is a good solution for EIS studies.

On the other hand, concerning the influence of the electrode materials (ITO, gold and platinum), we obtained a $R_{e t}$ around $20 \mathrm{k} \Omega$ for platinum, $45 \mathrm{k} \Omega$ for gold and $60 \mathrm{M} \Omega$ for ITO (data not shown). Since the smaller is the resistance of the electrode (before any treatment) the greater is its ability to detect any change due to the adhesion of cells, gold and platinum electrodes were preferred with respect to ITO and for this reason we have chosen to use for further experiments gold electrodes which can be also easily modified with different molecules and biomolecules (e.g. by thiol chemistry). In this case, to keep the full device (semi)transparent and maintain cells optically accessible to correlate impedimetric measurements with cell status, a very thin chromium/gold layer $(3 / 10 \mathrm{~nm})$ was deposited.

To test the ability of our chip to detect cells on its surface, we have used HeLa cells, an immortal cell line derived from human cervical cancer cells. Cell culture was carried out under standard conditions of $37^{\circ} \mathrm{C}$ and $5 \% \mathrm{CO}_{2}$ in air with Dulbecco's Modified Eagle's Medium (DMEM) (Sigma-Aldrich) supplemented with 10\% (v/ v) fetal bovine serum (FBS, Sigma-Aldrich), Sodium piruvate $1 \mathrm{mM}$ and with $100 \mathrm{~g} / \mathrm{ml}$ penicillin, $100 \mathrm{U} / \mathrm{ml}$ streptomycin, and $100 \mathrm{~g} / \mathrm{ml}$ L-glutamine. Before using the device we have investigated the tox- 


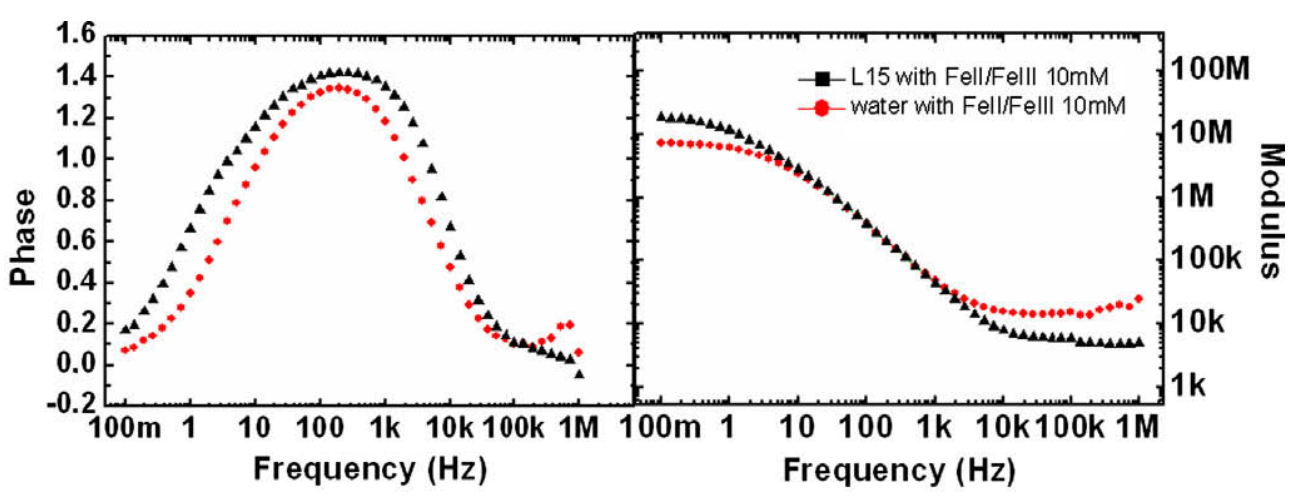

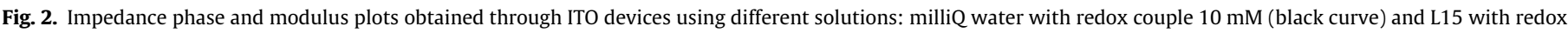
couple $10 \mathrm{mM}$ (red curve).

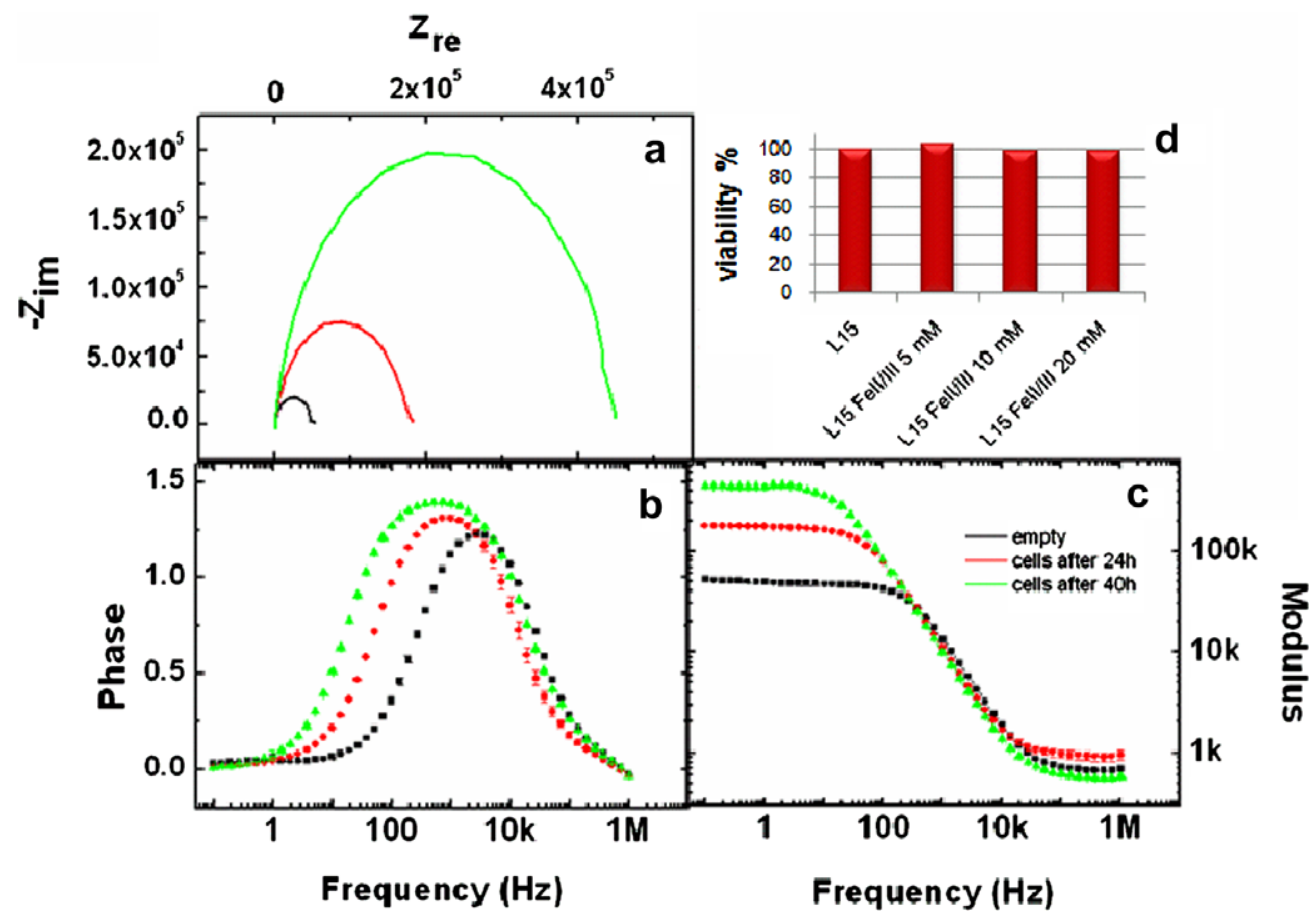

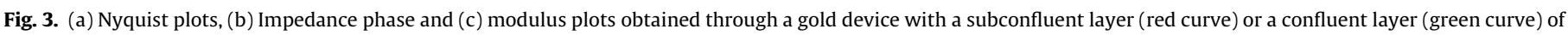

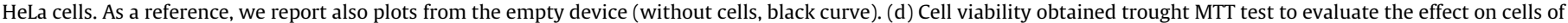
$\mathrm{K}_{3}\left[\mathrm{Fe}(\mathrm{CN})_{6}\right] / \mathrm{K}_{4}\left[\mathrm{Fe}(\mathrm{CN})_{6}\right]$ at different concentration.

icity of the redox couple on cells to exclude any influence from the medium. As shown in Fig. 3d the viability of cells is not affected by the presence of redox couple.

Beafore usage the chips were washed in ethanol before seeding and dried under a gentle nitrogen flow. It is even possible to sterilize the whole device by autoclave without any damage for it. HeLa cells resuspended in DMEM medium were seeded on each sensor chamber in a density of $3 \times 10^{5} \mathrm{cells} / \mathrm{ml}$ and then were allowed to grow for at least $24 \mathrm{~h}$.

Fig. 3 represents two different measurements on the same chip after cell growth: the red curve corresponds to the spectra of the cell after $24 \mathrm{~h}$ in culture while the green one represents the confluent cells after $40 \mathrm{~h}$ in culture. In the Nyquist plot the diameter of spectra increase depending on the number of cells, so the greater is the number of cells the greater is the interfacial resistance, demonstrating the ability of the device to detect different number of cells on its surface. Even in the modulus plot it's evident how at low frequencies (left side of the graph) the impedance value increases according to the number of cells onto the device (on the contrary high frequencies correlate with the impedance of medium and changes are minimal there).

\section{Conclusion}

Impedance measurements on cellular systems have been shown to be an effective tool for monitoring cellular behaviour. The results reveal that our EIS cell chips provide an easy and real-time monitoring tool to study cells attachment, spreading and to perform viability tests; adherent cells can be monitored during cultivation allowing us to study dynamic processes without any damage for cells. Our device is very cheap and reusable and it joins a great sensitivity and low cost both in fabrication and usage, in fact it does not require any additional reagent neither any pretreatment of the sensor. We expect that in the future such a chip can replace the conventional methods for cell counting, or for performing cytotoxicity tests thanks to its cheapness, fast response and ease of use as compared with traditional techniques. 


\section{References}

[1] C.R. Keese, I. Giaeverm, Proceedings of the National Academy of Sciences 88 (1991) 7896-7900

[2] C.R. Keese, I. Giaever, IEEE Engineering in Medicine and Biology Magazine 1994) 402-408.

[3] A. Bouafsoun et al., Macromolecular Biosciences 7 (2007) 599-610.

[4] J. Wegener et al., Experimental Cell Research 259 (2000) 158-166.

[5] J.H. Yeon, J.-K. Park, Analytical Biochemistry 341 (2005) 308-315.

[6] C. Xiao et al., Analytical Chemistry 74 (2002) 5748-5753.

[7] C. Xiao, J.H.T. Luong, Biotechnology Progress 19 (2003) 1000-1005.

[8] L. Ceriotti et al., Sensors and Actuators B: Chemical 123 (2) (2007) 769-778.
[9] R. Ehret et al., Biosensors and Bioelectronics 12 (1997) 29-41.

[10] S. Zheng et al., Biomed Microdevices 10 (2008) 221-223.

[11] L. Yang et al., Biosensors and Bioelectronics 19 (2004) 1139-1147.

[12] L. Yang et al., Analytical Chemistry 76 (4) (2004) 1107-1113.

[13] E. Katz, I. Willner, Electroanalysis 15 (2003) 913-947.

[14] X. Cheng et al., Lab on a Chip 7 (2007) 746-755.

[15] S. Arndt et al., Biosensors and Bioelectronics 19 (2004) 583-594.

[16] Y. Chen et al., Biosensors and Bioelectronics 23 (2008) 1390-1396.

[17] K.B. Male et al., Analytical Chemistry 80 (2008) 5487-5493.

[18] L. Balasubramanian et al., American Journal of Physilogy-Cell Physiology 295 (2008) C954-C965. 